\title{
Correlation of Bite Force Interpretation in Maximal Intercuspal Position among Patient, Clinician, and T-Scan III System
}

\author{
Nitikarn Ruttitivapanich ${ }^{1}$ Ratchawan Tansalarak ${ }^{2} \quad$ Jadesada Palasuk $^{3} \quad$ jittima Pumklin ${ }^{3}$ \\ ${ }^{1}$ Section of Orthodontics, Department of Preventive Dentistry, \\ Faculty of Dentistry, Naresuan University, Phitsanulok, Thailand \\ 2Department of Preventive Dentistry, Faculty of Dentistry, Naresuan \\ University, Phitsanulok, Thailand \\ Address for correspondence jittima Pumklin, DDS, MS, PhD, \\ Department of Restorative Dentistry, Faculty of Dentistry, Naresuan \\ University, Phitsanulok 65000, Thailand \\ (e-mail: Jittimap@nu.ac.th).
}

${ }^{3}$ Department of Restorative Dentistry, Faculty of Dentistry, Naresuan University, Phitsanulok, Thailand

\begin{abstract}
Keywords

- T-Scan

- bite force

- occlusal indicators

Objective The main purpose of this article was to determine the correlation of bite force in maximal intercuspal position (MIP) among patient's perceptions, clinician subjective interpretation, and T-Scan III system.

Materials and Methods Forty-three dental students at Naresuan University (Phitsanulok, Thailand) participated in the study. Subjects were positioned by Frankfurt horizontal plane paralleled to the horizontal plane and asked to bilaterally clenched in MIP. Patient's perception was evaluated by asking which side of the jaw had heavier bite force (right, left, or equally on both sides). Then, the clinician subjective interpretation was assessed using traditional occlusal indicators. Furthermore, patient's bite force was analyzed using T-Scan III.

Statistical Analysis Cohen's weighted kappa test was used to evaluate the correlation of bite force.

Results The best correlation between patient's perception and T-Scan III was at the $\pm 7.5 \%$ cutoff range with 15 subject agreements. While the best correlation between clinician subjective interpretation and T-Scan III was at $\pm 5.0 \%$ cutoff range with 23 subject agreements. Cohen's weighted kappa indicated slight agreement between T-Scan III and patient's perception and fair agreement between T-Scan III and clinician.

Conclusions Clinician subjective interpretation is more clinically reliable than patient's perception when T-Scan III is used as a gold standard.
\end{abstract}

\section{Introduction}

Perception of dental occlusion in human results from the sensory signal via proprioceptors. ${ }^{1-3}$ Even the pulpless teeth have similar sensory response upon loading ${ }^{4}$ compared with vital teeth as the periodontal mechanoreceptors play a significant role in bite force perception. ${ }^{1,3,5}$ The dental occlusion can not only be detected by patients but also be examined by clinicians via the use of various occlusal indicators.

Routine examination of occlusion is a combination of patient's perceptions and clinician. Clinicians interpret dental occlusion by evaluating the ink marks of articulating paper, pulling force of shim stock foil between a pair of occluded teeth, and translucent or perforation area of occlusal indicator wax. ${ }^{6}$ Although these traditional occlusal indicators were practical, their results are variable due to limited ability to discriminate dental occlusion. To overcome the limitations, digital occlusal indicators (i.e., T-Scan system) had been developed.

The T-Scan III system (Tekscan, Boston, Massachusetts, United States) is the newest version of the digital occlusal indicators. ${ }^{78}$ It could analyze and report occlusion in terms 
of the sequence of each tooth contact in 0.003 second time increments, the force location on the contacting tooth surfaces, the relative occlusal force in percentage values, and the center of force trajectory. ${ }^{7-9}$ Although the data from T-Scan III are more precise and quantitative than the conventional occlusal indicators, T-Scan III is not prevailingly used due to high system cost. Thus, patient's perceptions and clinician are still key factors to validate patient's occlusion.

Currently, no evidence demonstrated the accuracy of bite force from patient's perceptions or clinician compared with the T-Scan III. Herein, this study aimed to find the correlation of bite force in MIP among patient's perceptions, clinician subjective interpretation, and T-Scan III analysis.

\section{Materials and Methods}

This study was approved by Naresuan University Ethical Committee (IRB No.0995/60). The data were collected during April to June 2018. Forty-three undergraduate dental students at Naresuan University (Phitsanulok, Thailand) who had at least 24 permanent teeth with their same arch counterpart teeth were enrolled in this study. The subjects who presented with second- or third-degree tooth mobility, orthodontic treatment, temporomandibular disorder and parafunctional habit, or any dental treatment during duration of study were excluded.
Two appointments were planned in all subjects ( $\mathbf{- F i g . 1}$ ). In the first appointment, the dental occlusion was examined by occlusion specialized dentist (JP). The alginate impression for study models was done. The study models were used to measure mesiodistal width of teeth to set the subject's arch dimension in T-Scan III program. All subjects were repeatedly trained to bite at MIP.

One week later (the second appointment), the patient's perception, clinician subjective interpretation (JP), and T-Scan III analysis were collected. Subjects were requested to three times bilaterally clench their teeth at $\mathrm{MIP}^{10}$ as well as to report whether or not the teeth were occluded equally on both sides or which side was heavier. Second, JP used the occlusal indicator wax (Kerr, Orange, California, United States) to examine the size and force distribution of occlusion at MIP and the occlusal contacts were confirmed using shim stock foil (Hanel, Langenau, Germany). Then, JP would define the bite force (right, left, or equally on both sides). Lastly, to collect T-Scan III data, the subjects bilaterally clenched their teeth at the same MIP on the high definition (HD) sensor of T-Scan III three times. Relative bite force of right and left sides was reported as mean values and showed in percentage of each side. The cumulative relative bite force of both sides was $100 \%$. The different cutoff values $( \pm 2.5, \pm 5.0, \pm 7.5, \pm 10.0 \%$ ) deviated from $50 \%$ relative bite force were used to define the equal bite force of both right and left sides. For example, $\pm 10 \%$ cutoff

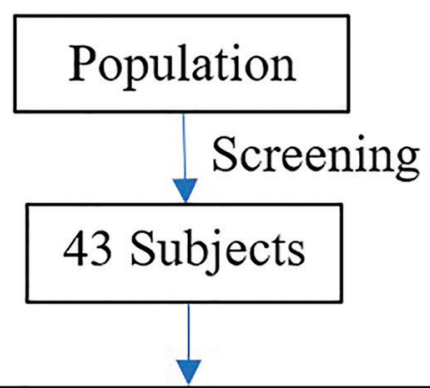

Visit 1

Visit 2

Data collection of - Patient's perception

- Clinician subjective interpretation

- T-Scan III analysis

Summary

Fig. 1 The flowchart of the study protocol. 

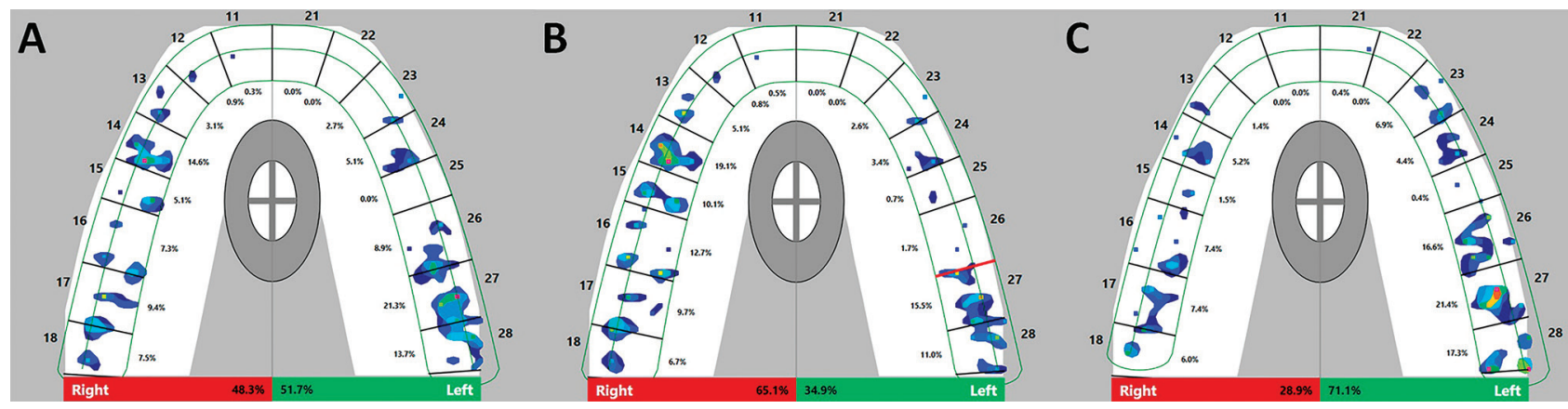

Fig. 2 The relative bite force is shown in T-Scan III display. When cutoff value was $\pm 10 \%$, equally bite force (A), right side was heavier than left side (B), and left side was heavier than right side (C).

value means that if relative bite force fell between 40 and $60 \%$, it would be interpreted as equal bite force of both right and left sides. If one side had relative bite force more than $60 \%$, it would be recognized as that side had heavier bite force than another one (-Fig. 2).

Sixteen subjects were randomly collected for intraexaminer reliability of clinician subjective interpretation at visit 1 and 2. To determine the correlation of bite force and intraexaminer reliability, Cohen's weighted kappa were calculated with SPSS Version 17.0 for Windows (SPSS Inc, Chicago, Illinois, United States). The kappa score was categorized as follows: poor (score $<0$ ), slight (score $0.00-0.20$ ), fair (score $0.21-0.40$ ), moderate (score $0.41-0.60$ ), substantial (score $0.61-0.80$ ), and almost perfect (score $0.81-1.00$ ) agreement. ${ }^{11}$

\section{Results}

Among 43 subjects, there were 29 females and 14 males with the age ranges between 19 and 23 years old. Bite force agreement between patient's perceptions and T-Scan III is shown in -Table 1. The maximum number of subject's perception corresponded with T-Scan III was 15 subjects when both sides cutoff values were \pm 7.5 . The least agreement (13 subjects) was observed when both sides cutoff values were \pm 2.5 .

- Table 2 demonstrated bite force agreement between clinician subjective interpretation and T-Scan III. The most bite force agreement was 23 subjects with both sides cutoff values of \pm 2.5 and \pm 5 .0. The least bite force agreement comprised of 17 subjects when both sides cutoff values were \pm 10.0 .

Cohen's weighted kappa indicated the correlation between patient's perceptions or clinician subjective interpretation

Table 1 Bite force agreement between patient's perceptions and T-Scan III at different cutoff values

\begin{tabular}{|c|c|c|c|}
\hline & & Number of & cts $(n=43)$ \\
\hline $\begin{array}{l}\text { Both si } \\
\text { (value }\end{array}$ & $\begin{array}{l}\text { es cutoff values } \\
\text { ange, \%) }\end{array}$ & Number & Subject \\
\hline \pm 2.5 & $(47.5-52.5 \%)$ & 13 & 30.23 \\
\hline \pm 5.0 & $(45.0-55.0 \%)$ & 14 & 32.56 \\
\hline \pm 7.5 & $(42.5-57.5 \%)$ & 15 & 34.88 \\
\hline \pm 10.0 & $(40.0-60.0 \%)$ & 14 & 32.56 \\
\hline
\end{tabular}

compared with T-Scan III as shown in - Table $\mathbf{3}$ and $\boldsymbol{- F i g} \mathbf{3}$. According to Landis and Koch, ${ }^{11}$ the best correlation between patient's perceptions and T-Scan III was slight agreement when cutoff value was \pm 7.5 and the best correlation between clinician and T-Scan III was fair agreement when cutoff value was \pm 5.0 .

Intraexaminer reliability of clinician was moderate agreement (12-16 subjects; kappa score 0.590 ) (data not show).

\section{Discussion}

This study demonstrated that the correlation between patient's perceptions and T-Scan III was slight agreement. This finding may be related to limited patient's recognition to the bite force. Siirilae and Laine stated that human can distinguish 8 to 10 microns thickness of foreign bodies between interocclusal surfaces. ${ }^{12}$ This could imply that subjects cannot recognize the true bite force when the interocclusal spaces

Table 2 Bite force agreement between clinician subjective interpretation and T-Scan III at different cutoff values

\begin{tabular}{|c|c|c|c|}
\hline \multirow{2}{*}{\multicolumn{2}{|c|}{$\begin{array}{l}\text { Both sides cutoff values } \\
\text { (value range, \%) }\end{array}$}} & \multicolumn{2}{|c|}{ Number of subjects $(n=43)$} \\
\hline & & \multirow{2}{*}{$\begin{array}{l}\text { Number } \\
\text { of subject } \\
\text { agreement } \\
23\end{array}$} & \multirow{2}{*}{$\begin{array}{l}\text { Subject } \\
\text { agreement } \\
\text { (\%) }\end{array}$} \\
\hline \pm 2.5 & $(47.5-52.5 \%)$ & & \\
\hline \pm 5.0 & $(45.0-55.0 \%)$ & 23 & 53.49 \\
\hline \pm 7.5 & $(42.5-57.5 \%)$ & 21 & 48.84 \\
\hline \pm 10.0 & $(40.0-60.0 \%)$ & 17 & 39.53 \\
\hline
\end{tabular}

Table 3 The Cohen's weighted kappa of each cutoff values

\begin{tabular}{|l|l|l|l|}
\hline \multirow{2}{*}{\multicolumn{2}{|l|}{$\begin{array}{l}\text { Both sides cutoff values } \\
\text { (value range, \%) }\end{array}$}} & \multicolumn{2}{|l|}{ Cohen's weighted kappa } \\
\cline { 3 - 4 } & $\begin{array}{l}\text { Patient's } \\
\text { perception }\end{array}$ & $\begin{array}{l}\text { Clinician } \\
\text { subjective } \\
\text { interpretation }\end{array}$ \\
\hline \pm 2.5 & $(47.5-52.5 \%)$ & -0.011 & 0.298 \\
\hline \pm 5.0 & $(45.0-55.0 \%)$ & -0.007 & 0.302 \\
\hline \pm 7.5 & $(42.5-57.5 \%)$ & 0.036 & 0.233 \\
\hline \pm 10.0 & $(40.0-60.0 \%)$ & 0.017 & 0.096 \\
\hline
\end{tabular}




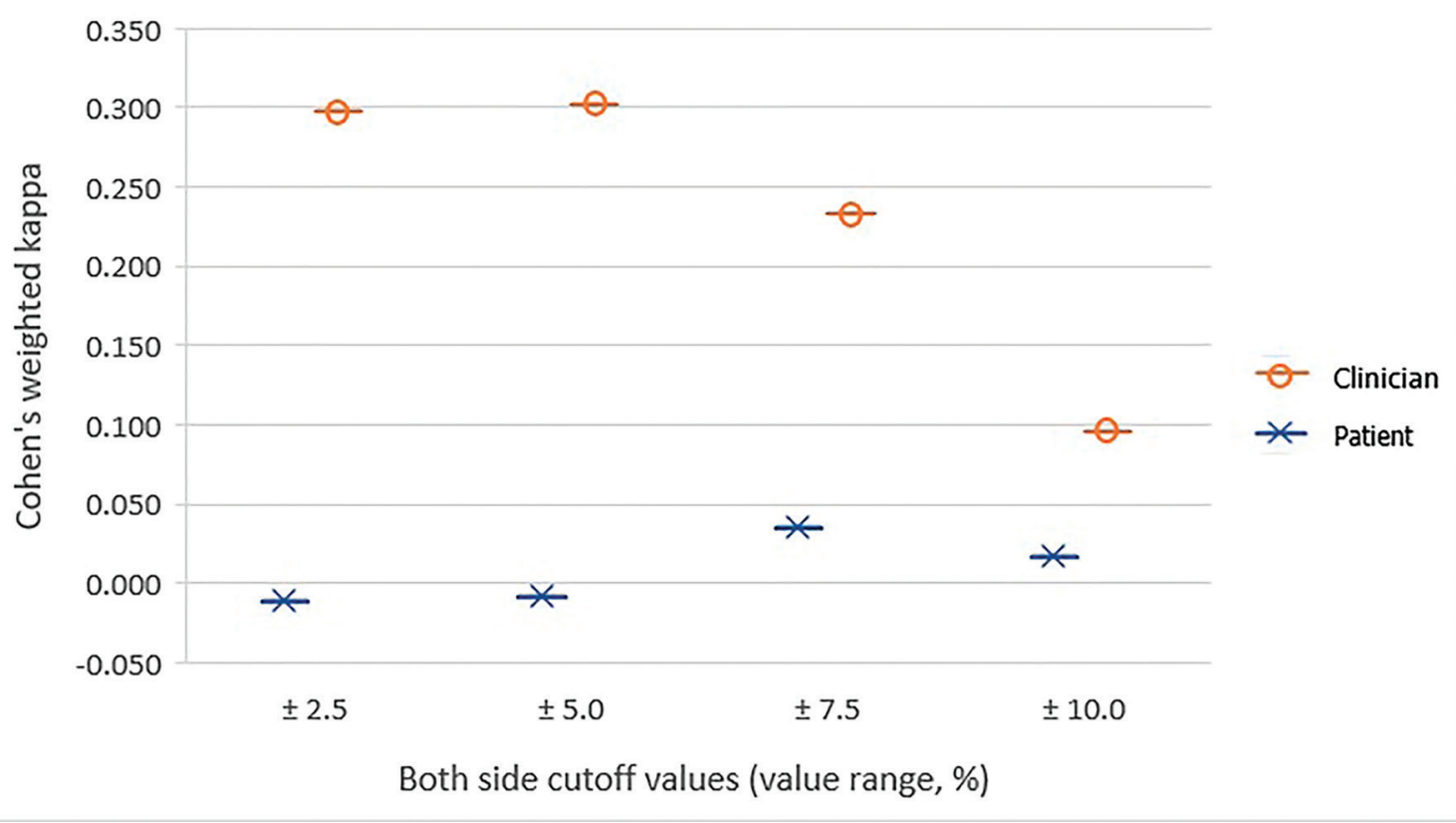

Fig. 3 The Cohen's weighted kappa of each cutoff values.

between occluding teeth at MIP are less than 8 to 10 microns. If these spaces are larger than 8 to 10 microns, patients would be able to differentiate which pairs of teeth had the heavier contacts without perceiving summation of the total force to differentiate teeth contacts.

Several studies investigated the bite force perception. ${ }^{13-18}$ Most of them evaluated single tooth or localized occlusal contacts, or before and after occlusal surface changes. ${ }^{17}$ Kampe et al found that the best occlusion perception was at the incisor regions, followed by canine and premolar regions, respectively ${ }^{16}$ This study evaluated the summation of occlusal force from patient's perception compared with T-Scan III that is served as a standard because of a more quantitative measurement than the aforementioned techniques. Our results showed slight agreement between patient's perceptions and T-Scan III which indicated that patient's bite force perception did not correlate well with the real bite force as measured by T-Scan III.

The effects of HD sensor should be taken into the consideration. In this study, the same HD sensor was used multiple times but less than 20 closures/subject as reported by Kerstein et $\mathrm{a}^{19}$ that consistency of the data from the HD sensor was not affected by up to 20 repeated closures. Although several investigators supported the efficacy and reliability of T-Scan III, ${ }^{7,19-21}$ the thickness of HD sensor (100 microns), which is $\sim 4$ to 12 times higher than physiologic vertical tooth movement (ca. 8-28 microns) in clinically healthy tooth, ${ }^{22}$ may interfere the patient biting into the same MIP. This may result in high variation in relative bite force of each subject. To overcome this problem, the average data of three repeated closures were used. Moreover, dental students who most likely know the biting pattern of their teeth were recruited in the study, instead of using general population. These could reduce the variability of each bite on HD sensor.
Even though clinical examination may be the most feasible and more reliable technique to examine tooth contact, no studies confirmed the accuracy of clinician subjective interpretation of bite force. Kerstein and Radke studied accuracy of clinician when interpreting articulating paper markings compared with T-Scan III. Their results indicated that the clinician did not effectively determine the relative bite force of patient. ${ }^{23}$ The intraexaminer reliability of JP was moderate agreement when traditional occlusal indicators were used. This may possibly due to the limited properties of the traditional occlusal indicators that could affect the clinician interpretation such as thickness, elasticity, and strength of the materials when they were used in the oral cavity. This can result in distortion/tearing of occlusal indicator wax/shim stock foil and may eventually produce false occlusal contacts ${ }^{6}$ as well as clinician's experience that should be considered. However, the correlation between clinician subjective interpretation and T-Scan III was fair agreement compared with slight agreement of patient's perception. It indicated that clinician is more dependable than patient's perception because of higher kappa score and narrower cutoff range. Lastly, further study is needed to demonstrate a correlation between T-Scan III and combined the results from patient's perception with clinician subjective interpretation for represent the clinical routine examination.

\section{Conclusion}

Clinician subjective interpretation was more consistent with T-Scan III when compared with patient's perception in term of occlusal force in MIP and, therefore, clinically dependable than patient's perception. T-Scan is used as a gold standard because it is a computerized occlusal analysis that is developed for quantitative clinical use. Additionally, data from patient's perception alone should be used with cautious especially for irreversible dental treatment. 


\section{Funding}

The Research Funds for Graduate Students from Faculty of Dentistry, Naresuan University.

\section{Conflict of Interest}

None declared.

\section{References}

1 Crum RJ, Loiselle RJ. Oral perception and proprioception: a review of the literature and its significance to prosthodontics. J Prosthet Dent 1972;28(2):215-230

2 Kobayashi M. Mechanisms of orofacial sensory processing in the rat insular cortex. J Oral Biosci 2018;60:59-64

3 Türker KS, Sowman PF, Tuncer M, Tucker KJ, Brinkworth RS. The role of periodontal mechanoreceptors in mastication. Arch Oral Biol 2007;52(4):361-364

4 Pfaffmann C. Afferent impulses from the teeth due to pressure and noxious stimulation. J Physiol 1939;97(2):207-219

5 Trulsson M. Force encoding by human periodontal mechanoreceptors during mastication. Arch Oral Biol 2007;52(4):357-360

6 Kerstein RB, Handbook of Research on Computerized Occlusal Analysis Technology Applications in Dental Medicine. Hershey: IGI Global; 2015

7 Cohen-Lévy J, Cohen N. Computerized occlusal analysis in dentofacial orthopedics: indications and clinical use the T-Scan III system. J Dentofacial Anom Orthod 2012;15:203-227

8 Kim J., Computerized Occlusion Using T-Scan III. South Boston, MA: Tekscan; 2014

9 Kerstein RB, Time-sequencing and force-mapping with integrated electromyography to measure occlusal parameters. In: Daskalaki A, ed. Clinical Technologies: Concepts, Methodologies, Tools and Applications. Hershey: IGI Global; 2010:88-110

10 Koc D, Dogan A, Bek B. Bite force and influential factors on bite force measurements: a literature review. Eur J Dent 2010;4(2):223-232

11 Landis JR, Koch GG. The measurement of observer agreement for categorical data. Biometrics 1977;33(1):159-174
12 Siirilae HS, Laine P. The tactile sensibility of the parodontium to slight axial loadings of the teeth. Acta Odontol Scand 1963;21:415-429

13 Alomari SA, Alhaija ES. Occlusal bite force changes during 6 months of orthodontic treatment with fixed appliances. Aust Orthod J 2012;28(2):197-203

14 Lundqvist S, Haraldson T. Occlusal perception of thickness in patients with bridges on osseointegrated oral implants. Scand. J Dent Res 1984;92(1):88-92

15 Hämmerle $\mathrm{CH}$, Wagner $\mathrm{D}$, Brägger $\mathrm{U}$, et al. Threshold of tactile sensitivity perceived with dental endosseous implants and natural teeth. Clin Oral Implants Res 1995;6(2):83-90

16 Kampe T, Haraldson T, Hannerz H, Carlsson GE. Occlusal perception and bite force in young subjects with and without dental fillings. Acta Odontol Scand 1987;45(2):101-107

17 Karlsson S, Molin M. Effects of gold and bonded ceramic inlays on the ability to perceive occlusal thickness. J Oral Rehabil 1995;22(1):9-13

18 Williams WN, Levin AC, LaPointe LL, Cornell CE. Bite force discrimination by individuals with complete dentures. J Prosthet Dent 1985;54(1):146-150

19 Kerstein RB, Lowe M, Harty M, Radke J. A force reproduction analysis of two recording sensors of a computerized occlusal analysis system. Cranio 2006;24(1):15-24

20 Lila-Krasniqi ZD, Shala KSh, Pustina-Krasniqi T, Bicaj T, Dula LJ, Guguvčevski L. Differences between centric relation and maximum intercuspation as possible cause for development of temporomandibular disorder analyzed with T-scan III. Eur J Dent 2015;9(4):573-579

21 Seth V, Patil AK, Kidiyoor H, Patil KT. Scan-An aid in achieving stable occlusion during finishing stages of orthodontic treatment. J Stomat Occ Med 2016;8:30-36

22 Hoffmann O, Zafiropoulos G-G. Tooth-implant connection: a review. J Oral Implantol 2012;38(2):194-200

23 Kerstein RB, Radke J. Clinician accuracy when subjectively interpreting articulating paper markings. Cranio 2014;32(1):13-23 